\title{
Stichwort: Abgrenzung
}

\author{
Marianne Eberhard-Kaechele
}

$\mathrm{n}$ der Behandlung von Menschen mit psychischen oder psychosomatischen Störungen wird oft auf eine mangelnde Abgrenzungsfähigkeit als Problem und eine Verbesserung der Abgrenzungsfähigkeit als Ziel Bezug genommen. Versucht man, diesen gängigen Begriff genau zu fassen, wird deutlich, dass es sich bei Abgrenzung um ein komplexes Phänomen handelt.

\section{Definitionen}

Eine Grenze ist laut Duden (2019a) a) die Limitierung von bzw. die Abschlusslinie oder Schranke gegenüber einem Phänomen, z.B. eine zeitliche Grenze, die Grenze des Machbaren, oder b) eine reale oder gedachte Trennungslinie zwischen unterschiedlichen Bereichen oder Erscheinungen, z.B. die Grenze zwischen Nord- und Süd-Korea, zwischen Kindheit und Jugend, zwischen Kunst und Kitsch.

Abgrenzen als Verb bedeutet, entsprechend der obigen Definition von Grenze a) etwas limitieren, begrenzen, zum Abschluss bringen, b) etwas oder sich durch genaue Bestimmung von etwas oder jemanden trennen, unterscheiden, c) sich von etwas distanzieren oder absetzen (Duden 2019b). Umschließt die Grenze ein Feld, kommt es zu Wirkungen wie Eingrenzen, Ausgrenzen und Angrenzen.

Im Kontext der Psychologie betrifft Abgrenzung die Fähigkeit, sich von anderen Menschen zu unterscheiden (Stern 2016), seine Bedürfnisse gegenüber anderen zu behaup- ten oder zu schützen (Wöller 2013; Sulz 2001) und seine Identität anhand seiner Ähnlichkeit und seiner Unterschiedlichkeit zu anderen zu definieren (Siegler et al. 2011). Die Fähigkeit, sich abzugrenzen, ist unmittelbar verknüpft mit und steht in dialektischer Beziehung zur Fähigkeit, sich einzulassen und zu verbinden (Bauriedl 2004; Petzold 2012).

\section{Abgrenzung im Laufe der Entwicklung}

Bei der Autonomieentwicklung über die Lebensspanne ist der Mensch mit Entwicklungsaufgaben konfrontiert, bei denen Abgrenzungsfähigkeit eine Rolle spielt und womöglich gestört werden kann.

1. Ab der Geburt ermöglicht Abgrenzung die Regulation bzw. den Schutz vor schädlichen Reizen, Überstimulation oder Überforderung durch Abwenden, Sich-Entfernen oder Widerstand-Leisten (Stern 1992).

2. Selbstgewahrsein entsteht durch die Unterscheidung von afferenter Wahrnehmung (Sinneseindrücke) und efferenter Wahrnehmung (motorischer Ausdruck/Handeln). Durch Vergleichsprozesse, Selbst- und Fremdzuschreibung und Selbstreflexion bildet sich die Identität des Kindes in Unterscheidung zu anderen aus (Siegler et al. 2011).

3. In frühkindlichen Interaktionen mit Bezugspersonen wechseln sich die Beteiligten idealerweise in ihrer Initiative, Einstimmung und ihrer Abgrenzung ab (Meltzoff/Moore 2006). 
Diese Verhaltensmuster bilden die Basis für soziale und Selbst-Regulation sowie die Reparatur von Interaktionsbrüchen, die gewöhnlicherweise in menschlichen Interaktionen auftreten (Shai / Fonagy 2013).

4. Die Entwicklung einer sicheren Bindung hängt von der Förderung der Abgrenzung und Verselbständigung des Kindes in der Exploration ab (Grossmann / Grossmann 2017).

5. In der Trotzphase macht das Kind große Fortschritte, und zwar a) motorisch: z. B. Fortbewegung, sich selbst füttern, mit Gegenständen funktionell umzugehen usw. und b) kognitiv / psychisch: z. B. eigene Ideen und Wünsche erproben sowie die Konzepte „ich“, „meins“, „nein“ und „stopp“ verstehen und selbst anwenden zur Eroberung und zum Schutz eigener Ressourcen (Sulz 2001).

6. Auf der Basis der Fähigkeit, eigene und fremde Gedanken, Bedürfnisse und Intentionen zu unterscheiden, erwirbt ein Kind die Kompetenzen zur Mentalisierung und Empathie (Shai / Fonagy 2013).

7. In der frühen Pubertät wird durch den Erwerb des abstrakten Denkens die Identität weiter ausgebaut, und die Unterscheidung von anderen bekommt einen besonderen Wert. Die verstärkte Auseinandersetzung mit der Meinung anderer verlangt eine besondere Balancierung von Abgrenzung und Anpassung, die in der späten Pubertät und im Erwachsenenalter idealerweise in eine integrierte und autonome Identität mündet (Siegler et al. 2011)

\section{Theoretische Modelle zu}

\section{Abgrenzungsfähigkeit und Abgrenzungsstörungen}

Bei einer sicheren Bindung zu den Eltern reifen die Eigenwilligkeit und Durchsetzungsfähigkeit als Ausdruck der Autonomieentwicklung in guter Balance zur Anpassungsfähigkeit und Sozialverhalten. Bei einer konflikthaften Entwicklung (durch Verbote, Ängstigung, starke
Kontrolle oder mangelnde Grenzen in Form von Vernachlässigung oder Verwöhnung) bleibt die Autonomieentwicklung unvollständig und führt zu spezifischen Verhaltensmustern im Hinblick auf Abgrenzung und Einlassen (Sulz 2001). Die Vielzahl der Theorien und Konstrukte, die sich mit dem Phänomen Abgrenzung befassen, teilen die Auffassung, dass Gesundheit sich durch eine situationsangepasste, flexible, dynamische und gemäßigte Unterscheidung von und Verbindung mit anderen Menschen auszeichnet. Pathologie äußert sich in Extremen, entweder der rigiden Abschottung oder der verstrickten Verbindung. Tabelle 1 gibt einen Überblick zu Theorien, die das Phänomen Abgrenzung betreffen.

Weitere verwandte Theorien sind u.a. die Merkmale Selbst-Objekt-Differenzierung und Bindungsfähigkeit der Strukturachse des OPD (Operationalisierte Psychodynamische Diagnostik, Arbeitskreis OPD 2014), das Abgrenzungsprinzip in der Therapie der Zweierbeziehung nach Willi (2012), Self-Expansion and Inclusion of Other in Self Theory (Aron et al. 2013) und Experiential Permeability (geringe oder hohe Offenheit für Erfahrungen) nach dem „Big Five“ der Persönlichkeitstheorie (Piedmont et al. 2009).

Exemplarisch wird hier die Theorie der Selbst-Andere-Differenzierung nach Bowen (1976) näher erläutert, die in Zeile 1 von Tabelle 1 erscheint. Abgrenzung als Selbst-AndereDifferenzierung ist eine Persönlichkeitseigenschaft, die zur psychologischen Reifung und Gesundheit notwendig ist. Abgrenzung beschreibt den Grad, zu dem eine Person rationales und emotionales Verhalten sowie Verbundenheit und Autonomie in Beziehungen differenzieren und regulieren kann. Skowron und Friedlander (1998) erläutern diese Parameter wie folgt:

Gut abgegrenzte oder differenzierte Personen können adaptiv wechseln zwischen emotionalen oder rationalen Funktionen, auch wenn die Umwelt emotional heftig reagiert. 
Tab. 1: Verwandte Konzeptionen von pathogenetischer und salutogenetischer Abgrenzung

\begin{tabular}{|c|c|c|c|}
\hline \multicolumn{4}{|l|}{ Bildmetapher } \\
\hline Theorie / Autoren & Gefährdet & Gesund & Gefährdet \\
\hline $\begin{array}{c}\text { Selbst-Andere- } \\
\text { Differenzierung } \\
\text { (Bowen } 1976 \\
\text { Skowron / Friedlander } \\
\text { 1998) }\end{array}$ & $\begin{array}{l}\text { „Fusion“ } \\
\text { Grenzüberschreitung } \\
\text { trifft auf fehlende } \\
\text { Abgrenzung }\end{array}$ & $\begin{array}{c}\text { Angrenzungs- \& } \\
\text { Abgrenzungsfähigkeit }\end{array}$ & $\begin{array}{c}\text { „Abkapseln“ } \\
\text { (Cut off) Rigides } \\
\text { Abgegrenzt-Sein ohne } \\
\text { Kontakt }\end{array}$ \\
\hline $\begin{array}{l}\text { Dialektische } \\
\text { Ambivalenztheorie } \\
\text { (Bauriedl 2004) }\end{array}$ & $\begin{array}{l}\text { „entweder-oder“ } \\
\text { Entweder ich be- } \\
\text { stimme oder du. } \\
\text { Unterwerfung, um } \\
\text { Sicherheitsbedürf- } \\
\text { nisse zu erfüllen }\end{array}$ & $\begin{array}{l}\text { „und“ bzw. } \\
\text { „sowohl-als-auch“ } \\
\text { Beide führen und fol- } \\
\text { gen. Lebenswünsche } \\
\text { und Sicherheitsbe- } \\
\text { dürfnis in Balance }\end{array}$ & $\begin{array}{l}\text { „weder-noch“ } \\
\text { Weder ich gebe dir } \\
\text { Input, noch will ich } \\
\text { von dir welches. } \\
\text { Rückzug, um Autono- } \\
\text { mie zu schützen }\end{array}$ \\
\hline $\begin{array}{c}\text { Körpergrenzen- } \\
\text { Konzept } \\
\text { (Cariola } 2015 \text { nach } \\
\text { Fisher / Cleveland } \\
\text { 1958) }\end{array}$ & $\begin{array}{l}\text { „Falsches Selbst“ } \\
\text { Ich lebe, was der } \\
\text { andere sehen will }\end{array}$ & $\begin{array}{l}\text { „Adaptives Selbst“ } \\
\text { Ich lebe einen Kom- } \\
\text { promiss zwischen } \\
\text { Wahrheit und sozial } \\
\text { Erwünschtem }\end{array}$ & $\begin{array}{c}\text { „Wahres Selbst“ } \\
\text { Ich lebe die Wahrheit } \\
\text { ohne Rücksicht auf } \\
\text { Verluste }\end{array}$ \\
\hline $\begin{array}{l}\text { Boundaries in the } \\
\qquad \text { Mind } \\
\text { (Grenzen im Geist) } \\
\text { (Hartmann 2011) }\end{array}$ & $\begin{array}{l}\text { Dünne, durchlässige, } \\
\text { flexible Grenzen }\end{array}$ & $\begin{array}{l}\text { Mittlere Ausprägung } \\
\text { von Grenzen }\end{array}$ & $\begin{array}{l}\text { Dicke, undurchlässige } \\
\text { rigide Grenzen }\end{array}$ \\
\hline $\begin{array}{l}\text { Bindungstheorie im } \\
\text { Erwachsenenalter } \\
\text { (Main et al. 1985) }\end{array}$ & $\begin{array}{c}\text { Präokkupierte, } \\
\text { verstrickte Bindungs- } \\
\text { einstellung }\end{array}$ & $\begin{array}{l}\text { Autonome Bindungs- } \\
\text { einstellung }\end{array}$ & $\begin{array}{l}\text { Distanziert-bezie- } \\
\text { hungsabweisende } \\
\text { Bindungseinstellung }\end{array}$ \\
\hline
\end{tabular}

Sie haben eine eigene Meinung und einen stabilen Sinn für die eigene Identität. Aus diesem Grund können sie in zwischenmenschlichen Beziehungen flexibel und adaptiv sowohl mit der Nähe und Intimität in der Angrenzung als auch Distanz und Autonomie in der Abgrenzung umgehen. Sie können ihre eigenen Verhaltensziele beibehalten, auch unter Einfluss von der sozialen Umwelt.

Wenig abgegrenzte Personen reagieren stark auf die Emotionalität anderer (emotional reactivity). Sie neigen dazu, entweder mit anderen zu verschmelzen (fusion) oder sich von anderen abzukapseln (cut-off). Sie übernehmen die Gedanken, Gefühle und Verhalten an- derer (verschmelzen / fusion), oder sie wirken emotional unbeteiligt, lassen sich von nichts beeindrucken und beharren dogmatisch auf bestimmten Überzeugungen oder Verhaltensweisen (abkapseln / cut-off). Wenig abgegrenzte Personen neigen dazu, situationsunabhängig entweder klammernd in Nähe zu verharren und Disharmonie und Trennung zu fürchten (fusion), oder sie halten sich ständig auf Distanz mit demonstrativer Autonomie und fürchten Nähe (cut-off). In beiden Fällen reagieren sie empfindlich gegenüber dem Einfluss der sozialen Umwelt, und zwar gemäß ihrem Verhaltensmuster unterwürfig bzw. rebellisch. Sie sind weniger stressresistent als gut abgegrenzte 
Personen und daher anfällig für psychische und psychosomatische Störungen.

$\mathrm{Zu}$ den Störungen, die mit mangelnder Abgrenzungs- / Angrenzungsbalance assoziiert werden, gehören Depression, Angststörungen, Somatisierungsstörungen, Süchte, Psychosen, Essstörungen, PTBs und Zwänge (Hartmann 2011; Skowron / Friedlander 1998; Sulz 2001; Wöller 2013).

\section{Facetten der Abgrenzung}

Zum Abschluss folgt eine Liste von Facetten der Abgrenzung, die in der Psychotherapie benannt wurden (Hartmann 2011; Sulz 2001; Wöller 2013) und die als Anregung für körperund bewegungsbezogene Interventionen dienen können:

1. Körpergrenzen: zwischen dem Körper und der Umwelt, die Hautoberfläche als Grenze, Körperschema, Körperöffnungen öffnen und schließen

2. Leistungs-/ Belastungsgrenzen: richtig einschätzen vs. Über- oder Unterschreitung

3. Kognitive Konzentration: sich von Störungen abgrenzen

4. Räumliche Grenzen: Nähe und Distanz, persönlicher Raum, Territorium, Besitz

5. Schutz eigener Bedürfnisse und Ressourcen durch Grenzsetzung: Nein sagen

6. Identität: Was unterscheidet mich von, was verbindet mich mit anderen?

7. Interpersönliche Grenzen: folgen, führen und allein sein, spiegeln und kontrastieren, Einlassen und Abgrenzung, Empathie und bei sich bleiben, Einstimmen und sein Gefühl oder Überzeugung trotz Widerstand beibehalten

8. Soziale Abgrenzung, Eingrenzung, Ausgrenzung in Paaren und Gruppen

9. Sicherheits- und moralische Grenzen: Regeln aufstellen, befolgen, sich widersetzen

10. Grenzen zwischen Zuständen und Phänomenen setzen / auflösen: träumen / wachen, kindlich / erwachsen, Realität / Imagination oder Erinnerung, Ratio / Emotion usw.

11. Der entscheidende Hinweis für die therapeutische Praxis ist, dass Abgrenzung nicht isoliert von der Angrenzung bzw. dem Einlassen gefördert werden sollte, sondern dass eine Balance zwischen beiden Modalitäten erst Stabilität und seelische Gesundheit ermöglicht.

\section{Literatur}

Arbeitskreis OPD (Hrsg.) (2014): OPD-2. Operationalisierte Psychodynamische Diagnostik.

3. überarb. Aufl. Huber, Bern

Aron, A., Lewandowski, G., Mashek, D., Aron, E. (2013): The self-expansion model of motivation and cognition in close relationships. In: Simpson, J., Campbell L. (Hrsg.): The Oxford handbook of close relationships. Oxford University Press, Oxford, 90-115, https://doi.org/ 10.1093/oxfordhb/9780195398694.013.0005

Bauriedl, T. (2004): Auch ohne Couch. Psychoanalyse als Beziehungstheorie und ihre Anwendungen. Klett-Cotta, Stuttgart

Bowen, M. (1976): Theory in the practice of psychotherapy. In: Guerin Jr., P. (Hrsg.): Family therapy: Theory and practice. Garner Press, New York, 42-90

Cariola, L. (2015) Semantic expressions of the body boundary personality in person-centred psychotherapy. International Body Psychotherapy Journal 14 (1), 48-64

Duden (2019a): Grenze. In: www.duden.de/ rechtschreibung/Grenze, 4.12.2019, https://doi.org/10.1007/978-1-4684-7341-4

Duden (2019b): abgrenzen. In: www.duden.de/ rechtschreibung/abgrenzen, 4.12.2019, https://doi.org/10.1007/978-1-4684-7341-4

Fisher S., Cleveland, S. (1958): Body image and personality. Dover Publications, New York

Grossmann, Kl., Grossmann, Ka. (2017): Bindungen - das Gefüge psychischer Sicherheit. 7. Aufl. Klett-Cotta, Stuttgart

Hartmann, E. (2011): Boundaries. A new way to look at the world. CIRCC EverPress, Summerland

Main, M., Kaplan, N., Cassidy, J. (1985): Security in infancy, childhood, and adulthood: A move to the level of representation. In: Growing Points of 
Attachment Theory and Research 50 (1/2), 66104, https://doi.org/10.2307/3333827

Meltzoff, A., Moore, M. K. (2006): Infant intersubjectivity: broadening the dialogue to include imitation, identity and intention. In: Bråten S. (Hrsg.): Intersubjective communication and emotion in early ontogeny. Cambridge University Press, Cambridge, 47-62

Petzold, H.G. (2012): Psychotherapie - Arbeitsbündnis oder „Sprache der Zärtlichkeit“ und gelebte Konvivialität? Intersubjektive Nahraumbeziehungen als Prozesse affilialer „Angrenzung“ statt abgrenzender „Arbeitsbeziehungen“. Integrative Therapie 1, 73-94. In: www.fpipublikation.de/artikel/textarchiv-h-g-petzold-etal-/petzold-h-g-2012c-psychotherapie-arbeits buendnis-oder-sprache-der-zaertlichkeit-und. html, 14.12.2019, https://doi.org/10.30820/ 9783837968965-297

Piedmont, R., Sherman, M., Sherman, N., Dy-Liacco, G., Williams, J. (2009): Using the five-factor model to identify a new personality disorder domain: The case for experiential permeability. Journal of Personality and Social Psychology 96 (6), 1245-1258, https://doi.org/10.1037/ a0015368

Shai, D., Fonagy, P. (2013): Beyond words: Parental embodied mentalizing and the parent-infant dance. In: Mikulincer, M., Shaver P. (Hrsg.): Mechanisms of social connection: From brain to group. American Psychological Association, Washington, D.C., 185-203, https://doi.org/ 10.1037/14250-011

Siegler, R., DeLoache, J., Eisenberg, N. (2011): Entwicklungspsychologie im Kindes- und Jugendalter. Spektrum / Springer, Heidelberg, https://doi.org/10.1007/978-3-662-47028-2

Skowron, E. A., Friedlander, M. L. (1998): The differentiation of self inventory. Development and initial validation. Journal of Counseling Psychology 45 (3), 235-246, https://doi. org/10.1037//0022-0167.45.3.235

Stern, D. 2016): Die Lebenserfahrung des Säuglings. 11. Aufl. Klett-Cotta, Stuttgart

Sulz, S. (2001): Von der Strategie des Symptoms zur Strategie der Therapie: Gestaltung von Prozess und Inhalt in der Psychotherapie. CIP-Medien, München

Willi, J. 2012): Die Zweierbeziehung. Erw. Neuausg. Rowohlt, Reinbek

Wöller, W. (2013): Sich schützen und sich abgrenzen. In: Wöller, W., Reddemann L. (Hrsg.): Trauma und Persönlichkeitsstörungen. 2. überarb. Aufl. Schattauer, Stuttgart, 391-397

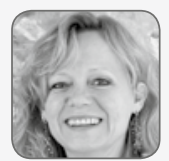

\section{Dr. rer. medic. Marianne} Eberhard-Kaechele

Ausbilderin, Supervisorin und Lehrtherapeutin BTD. Dozentin an der Deutschen Sporthochschule Köln am Institut für Bewegungstherapie und bewegungsorientierte Prävention und Rehabilitation. Eigene Praxis für Tanzund Ausdruckstherapie.

Dr. rer. medic. Marianne EberhardKaechele

Abteilung Neurologie, Psychosomatik, Psychiatrie Institut für Bewegungstherapie und bewegungsorientierte Prävention und Rehabilitation

Deutsche Sporthochschule Köln Am Sportpark Müngersdorf 6 | D-50933 Köln m.eberhard-kaechele@dshs-koeln.de 